\title{
Detecting structural properties of finite groups by the sum of element orders
}

\author{
Marius Tărnăuceanu
}

April 6, 2019

\begin{abstract}
In this paper, we introduce a new function related to the sum of element orders of finite groups. It is used to give some criteria for a finite group to be cyclic, abelian, nilpotent, supersolvable and solvable, respectively.
\end{abstract}

MSC2000 : Primary 20D60; Secondary 20D10, 20D15, 20F16, $20 \mathrm{~F} 18$.

Key words : group element orders, cyclic groups, abelian groups, nilpotent groups, supersolvable groups, solvable groups.

\section{Introduction}

Given a finite group $G$, we consider the function

$$
\psi(G)=\sum_{x \in G} o(x)
$$

where $o(x)$ denotes the order of $x$. This has been introduced by H. Amiri, S.M. Jafarian Amiri and I.M. Isaacs [1]. They proved the following theorem:

Theorem A. If $G$ is a group of order $n$, then $\psi(G) \leq \psi\left(C_{n}\right)$, and we have equality if and only if $G$ is cyclic.

In other words, the cyclic group $C_{n}$ is the unique group of order $n$ which attains the maximal value of $\psi(G)$ among groups of order $n$. 
Since then many authors have studied the function $\psi(G)$ and its relations with the structure of $G$ (see e.g. [2]-[5], [7]-[10], [12] and [14]). In the papers [4] and [12] M. Amiri and S.M. Jafarian Amiri, and, independently, R. Shen, $\mathrm{G}$. Chen and $\mathrm{C}$. Wu started the investigation of groups with the second largest value of the sum of element orders. M. Herzog, P. Longobardi and M. Maj [7] determined the exact upper bound for $\psi(G)$ for non-cyclic groups of order $n$ :

Theorem B. If $G$ is a non-cylic group of order $n$ and $q$ is the least prime divisor of the order of $n$, then

$$
\psi(G) \leq \frac{\left[\left(q^{2}-1\right) q+1\right](q+1)}{q^{5}+1} \psi\left(C_{n}\right)=f(q) \psi\left(C_{n}\right) .
$$

Moreover, the equality holds if and only if $n=q^{2} m$ with $(m, q !)=1$ and $G \cong\left(C_{q} \times C_{q}\right) \times C_{m}$.

Note that the above function $f$ is strictly decreasing on $[2, \infty)$. Consequently, we have

$$
\psi(G) \leq f(2) \psi\left(C_{n}\right)=\frac{7}{11} \psi\left(C_{n}\right),
$$

and the equality holds for $n=4 m$ with $m$ odd and $G \cong\left(C_{2} \times C_{2}\right) \times C_{m}$.

By using the sum of element orders, several criteria for solvability of finite groups have been determined (see e.g. [5, 8]). We recall here the following theorem of M. Baniasad Asad and B. Khosravi [5]:

Theorem C. If $G$ is a group of order $n$ and $\psi(G)>\frac{211}{1617} \psi\left(C_{n}\right)$, then $G$ is solvable.

Note that the equality $\psi(G)=\frac{211}{1617} \psi\left(C_{n}\right)$ occurs for $n=60 m$ with $(30, m)=1$ and $G \cong A_{5} \times C_{m}$.

We also recall a criterion for nilpotency of finite groups that has been proved in [14]:

Theorem D. If $G$ is a group of order $n$ and $\psi(G)>\frac{13}{21} \psi\left(C_{n}\right)$, then $G$ is nilpotent. Moreover, we have $\psi(G)=\frac{13}{21} \psi\left(C_{n}\right)$ if and only if $n=6 \mathrm{~m}$ with $(6, m)=1$ and $G \cong S_{3} \times C_{m}$.

The largest four values of the ratio $\psi^{\prime}(G)=\frac{\psi(G)}{\psi\left(C_{|G|}\right)}$ and the groups $G$ for which they are attained can be obtained from Theorem D.

Corollary E. Let $G$ be a finite group satisfying $\psi^{\prime}(G)>\frac{13}{21}$. Then $\psi^{\prime}(G) \in$ $\left\{\frac{27}{43}, \frac{7}{11}, 1\right\}$, and one of the following holds: 
a) $G \cong Q_{8} \times C_{m}$, where $m$ is odd;

b) $G \cong\left(C_{2} \times C_{2}\right) \times C_{m}$, where $m$ is odd;

c) $G$ is cyclic.

The above results show that a finite group $G$ becomes cyclic, abelian, nilpotent or solvable if $\psi^{\prime}(G)$ is sufficiently larget. In what follows, we consider the function

$$
\psi^{\prime \prime}(G)=\frac{\psi(G)}{|G|^{2}} .
$$

Clearly, $\psi^{\prime \prime}(G)<1$ if $G$ is non-trivial, and there are sequences of groups $\left(G_{n}\right)$ such that $\psi^{\prime \prime}\left(G_{n}\right)$ tends to 1 when $n$ tends to infinity (for example, $\left(C_{p}\right)$ where $p$ runs over the set of primes). We also observe that $\psi^{\prime \prime}$ satisfies the following important property

$$
\psi^{\prime \prime}(G) \leq \psi^{\prime \prime}(G / H), \forall H \triangleleft G,
$$

by Proposition 2.6 of [8]. We will use this new function to give criteria for a finite group to be cyclic, abelian, nilpotent, supersolvable and solvable, respectively. Our main result is the following theorem.

Theorem 1.1. Let $G$ be a finite group. Then the following hold:

a) If $\psi^{\prime \prime}(G)>\frac{7}{16}=\psi^{\prime \prime}\left(C_{2} \times C_{2}\right)$, then $G$ is cyclic;

b) If $\psi^{\prime \prime}(G)>\frac{27}{64}=\psi^{\prime \prime}\left(Q_{8}\right)$, then $G$ is abelian;

c) If $\psi^{\prime \prime}(G)>\frac{13}{36}=\psi^{\prime \prime}\left(S_{3}\right)$, then $G$ is nilpotent;

d) If $\psi^{\prime \prime}(G)>\frac{31}{144}=\psi^{\prime \prime}\left(A_{4}\right)$, then $G$ is supersolvable;

e) If $\psi^{\prime \prime}(G)>\frac{211}{3600}=\psi^{\prime \prime}\left(A_{5}\right)$, then $G$ is solvable.

Note that the converses of the implications in Theorem 1.1 are not true. We observe that $\psi^{\prime \prime}\left(C_{p^{m}}\right)$ tends to $\frac{p}{p+1}$ when $m$ tends to infinity. Let $\left(p_{i}\right)_{i \in \mathbb{N}^{*}}$ be the sequence of primes. Since $\prod_{i \geq 1}^{p+1} \frac{p_{i}}{p_{i}+1}=0$, there exists a positive integer $k$ such that $c=\prod_{i=1}^{k} \frac{p_{i}}{p_{i}+1}<\frac{211}{3600}$. If $n=\prod_{i=1}^{k} p_{i}^{n_{i}}$, then $\psi^{\prime \prime}\left(C_{n}\right)$ tends to $c$ when $n_{1}, \ldots, n_{k}$ tend to infinity. In other words, $\psi^{\prime \prime}\left(C_{n}\right)<\frac{211}{3600}$ for $n_{1}, \ldots, n_{k}$ sufficiently large, i.e. there are cyclic groups $G$ with $\psi^{\prime \prime}(G)<\frac{211}{3600}$.

\footnotetext{
${ }^{1} \mathrm{~A}$ similar result for supersolvability has been conjectured in [14].
} 
For the proof of the above theorem, we need some preliminary results about the function $\psi$ taken from [2, 7].

Lemma 1.2. Here $G$ denotes a finite group, $p, p_{i}$ denote primes and $n, n_{i}$, denote positive integers. The following statements hold:

1) ([7], Lemma 2.9(1)) $\psi\left(C_{p^{n}}\right)=\frac{p^{2 n+1}+1}{p+1}$;

2) ([7], Lemma 2.2(3)) $\psi$ is multiplicative, that is if $G=A \times B$, where $A, B$ are subgroups of $G$ satisfying $\operatorname{gcd}(|A|,|B|)=1$, then $\psi(G)=$ $\psi(A) \psi(B)$;

3) ([2], Lemma 2.1) $\psi(A \times B) \leq \psi(A) \psi(B)$. Moreover, $\psi(A \times B)=$ $\psi(A) \psi(B)$ if and only if $\operatorname{gcd}(|A|,|B|)=1$;

4) ([7], Lemma 2.9(2)) If $n=\prod_{i=1}^{k} p_{i}^{n_{i}}$, where $p_{i} \neq p_{j}$ for $i \neq j$, then $\psi\left(C_{n}\right)=\prod_{i=1}^{k} \psi\left(C_{p_{i}^{n_{i}}}\right)$;

5) ([7], Lemma 2.2(5)) If $G=P \rtimes H$, where $P$ is a cyclic p-group, $|H|>1$ and $(p,|H|)=1$, then $\psi(G)=|P| \psi(H)+(\psi(P)-|P|) \psi\left(C_{H}(P)\right)$.

We also need the following two theorems. The first one is due to A. Lucchini (see Theorem 2.20 in [6]), while the second one is a consequence of a theorem of B. Huppert and N. Ito (see Theorem 13.10.1 in [11]).

Theorem 1.3. Let $A$ be a cyclic proper subgroup of a finite group $G$, and let $K=\operatorname{Core}_{G}(A)$. Then $[A: K]<[G: A]$, and in particular, if $|A| \geq[G: A]$, then $K>1$.

Theorem 1.4. Suppose that a finite group $G$ contains a subgroup $B$ of prime power index and $B$ contains a cyclic subgroup $H$ of index $[B: H] \leq 2$. Then $G$ is solvable.

We end our paper by indicating a natural open problem concerning the criteria in Theorem 1.1.

Open problem. Determine all finite groups $G$ for which $\psi^{\prime \prime}(G)$ takes the values $\frac{7}{16}, \frac{27}{64}, \frac{13}{36}, \frac{31}{144}$ and $\frac{211}{3600}$, respectively.

Note that, given $c \in(0,1) \cap \mathbb{Q}$, the main difficulty in solving this problem is to determine positive integers $n$ such that $\psi^{\prime \prime}\left(C_{n}\right)=c$. 


\section{Proofs of the main results}

First of all, we give three lemmas that will be useful to us.

Lemma 2.1. Let $G$ be a finite group. If $\psi^{\prime \prime}(G) \geq \frac{1}{3}$, then either $G$ is cyclic or there exists $x \in G$ such that $[G:\langle x\rangle]=2$.

Proof. From the condition $\psi^{\prime \prime}(G) \geq \frac{1}{3}$ we infer that there exists $x \in G$ such that $o(x)>\frac{|G|}{3}$. This leads to $[G:\langle x\rangle]<3$, that is $[G:\langle x\rangle] \in\{1,2\}$. Then either $G=\langle x\rangle$ is cyclic or $[G:\langle x\rangle]=2$, as desired.

Lemma 2.2. Let $G$ be a finite 2-group having a cyclic maximal subgroup. Then the following hold:

a) If $\psi^{\prime \prime}(G)>\frac{7}{16}$, then $G$ is cyclic;

b) If $\psi^{\prime \prime}(G)>\frac{27}{64}$, then $G$ is cyclic or $G \cong C_{2} \times C_{2}$;

c) If $\psi^{\prime \prime}(G)>\frac{13}{36}$, then $G$ is cyclic or $G \cong C_{2} \times C_{2}$ or $G \cong Q_{8}$.

Proof. Assume that $G$ is not cyclic and let $n=|G|$. Then, by Theorem 4.1 of [13], II, we infer that either $G$ is abelian of type $C_{2} \times C_{2^{n-1}}, n \geq 2$, or non-abelian of one of the following types:

- $M\left(2^{n}\right)=\left\langle x, y \mid x^{2^{n-1}}=y^{2}=1, y x y=x^{2^{n-2}+1}\right\rangle, n \geq 4 ;$

- $D_{2^{n}}=\left\langle x, y \mid x^{2^{n-1}}=y^{2}=1, y x y=x^{-1}\right\rangle, n \geq 3$;

- $Q_{2^{n}}=\left\langle x, y \mid x^{2^{n-1}}=y^{4}=1, y x y^{-1}=x^{2^{n-1}-1}\right\rangle, n \geq 3 ;$

- $S_{2^{n}}=\left\langle x, y \mid x^{2^{n-1}}=y^{2}=1, y x y=x^{2^{n-2}-1}\right\rangle, n \geq 4$.

If $G \cong C_{2} \times C_{2^{n-1}}$, then

$$
\psi^{\prime \prime}(G)=\frac{2^{2 n}+5}{3 \cdot 2^{2 n}}>\frac{13}{36} \Leftrightarrow 2^{2 n}<60 \Leftrightarrow n=2 \text {, i.e. } G \cong C_{2} \times C_{2},
$$

while if $G$ is non-abelian, then we get:

$$
\begin{aligned}
& \text { - } \psi^{\prime \prime}\left(M\left(2^{n}\right)\right)=\frac{1}{2^{n}}<\frac{13}{36}, \forall n \geq 4 \\
& \text { - } \psi^{\prime \prime}\left(D_{2^{n}}\right)=\frac{2^{2 n-1}+3 \cdot 2^{n}+1}{3 \cdot 2^{2 n}}<\frac{13}{36}, \forall n \geq 3
\end{aligned}
$$




$$
\begin{aligned}
& \text { - } \psi^{\prime \prime}\left(Q_{2^{n}}\right)=\frac{2^{2 n-1}+3 \cdot 2^{n+1}+1}{3 \cdot 2^{2 n}}>\frac{13}{36} \Leftrightarrow n=3, \text { i.e. } G \cong Q_{8} ; \\
& \text { - } \psi^{\prime \prime}\left(S_{2^{n}}\right)=\frac{2^{2 n-1}+9 \cdot 2^{n-1}+1}{3 \cdot 2^{2 n}}<\frac{13}{36}, \forall n \geq 4 .
\end{aligned}
$$

This completes the proof.

Lemma 2.3. Let $G$ be a non-trivial semidirect product of $C_{p^{n}}$ by $C_{2}$, where $p$ is an odd prime and $n$ is a positive integer. Then $\psi^{\prime \prime}(G) \leq \frac{13}{36}$, and the equality occurs if and only if $p=3$ and $n=1$, i.e. $G \cong S_{3}$.

Proof. Lemma 1.2, 5), shows that

$$
\psi(G)=\psi\left(C_{p^{n}} \rtimes C_{2}\right)=p^{n} \psi\left(C_{2}\right)+\left(\psi\left(C_{p^{n}}\right)-p^{n}\right) \psi\left(C_{C_{2}}\left(C_{p^{n}}\right)\right) .
$$

Since the semidirect product $C_{p^{n}} \rtimes C_{2}$ is non-trivial, we have $C_{C_{2}}\left(C_{p^{n}}\right)=1$ and so

$$
\psi(G)=3 \cdot p^{n}+\frac{p^{2 n+1}+1}{p+1}-p^{n}=\frac{p^{2 n+1}+2 \cdot p^{n+1}+2 \cdot p^{n}+1}{p+1},
$$

i.e.

$$
\psi^{\prime \prime}(G)=\frac{p^{2 n+1}+2 \cdot p^{n+1}+2 \cdot p^{n}+1}{4\left(p^{2 n+1}+p^{2 n}\right)} .
$$

Now, the inequality $\psi^{\prime \prime}(G) \leq \frac{13}{36}$ is equivalent with

$$
4 \cdot p^{2 n+1}+13 \cdot p^{2 n}-18 \cdot p^{n+1}-18 \cdot p^{n}-9 \geq 0 .
$$

We easily observe that the following function on the real variable

$$
f(x)=4 \cdot x^{2 n+1}+13 \cdot x^{2 n}-18 \cdot x^{n+1}-18 \cdot x^{n}-9
$$

is strictly increasing on $[3, \infty)$. Consequently,

$$
f(x) \geq f(3)=25 \cdot 3^{2 n}-72 \cdot 3^{n}-9 \geq 25 \cdot 3^{n+1}-72 \cdot 3^{n}-9=3^{n+1}-9 \geq 0
$$

and we have equality if and only if $x=3$ and $n=1$, completing the proof.

We are now able to prove our main result.

Proof of Theorem 1.1. We first prove item c). Since $\psi^{\prime \prime}(G) \geq \frac{13}{36} \geq \frac{1}{3}$, by Lemma 2.1 it follows that either $G$ is cyclic or there exists $x \in G$ such that $[G:\langle x\rangle]=2$. Let $n=|G|=2^{n_{1}} p_{2}^{n_{2}} \cdots p_{k}^{n_{k}}$, where $p_{2}<p_{3}<\cdots<p_{k}$ are odd primes. Then, for each $i=2, \ldots, k,\langle x\rangle$ contains a cyclic Sylow $p_{i}$-subgroup $P_{i}$ of $G$. We have $\langle x\rangle \leq N_{G}\left(P_{i}\right)$ and so $P_{i}$ is normal in $G$. Therefore $G$ has a cyclic normal 2-complement, that is 


$$
G=C_{m} \rtimes H
$$

where $m=\frac{n}{2^{n_{1}}}$ is odd and $H$ is a Sylow 2-subgroup of $G$. Note that $\langle x\rangle$ also contains a cyclic normal subgroup $M$ of order $2^{n_{1}-1}$, that is $H$ possesses a cyclic maximal subgroup. Assume that $G$ is not nilpotent. We will show that $\psi^{\prime \prime}(G) \leq \frac{13}{36}$, contradicting our hypothesis. We infer that there exists

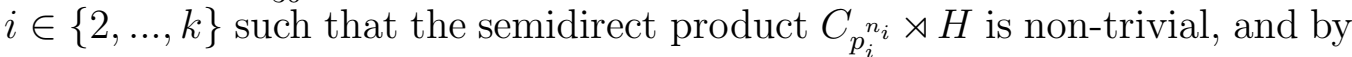
property (1) we may assume that $G=C_{p_{i}{ }_{i}} \rtimes H$. Then $G / M$ is a non-trivial semidirect product of $C_{p_{i} n_{i}}$ by $C_{2}$, and (1) and Lemma 2.3 imply that

$$
\psi^{\prime \prime}(G) \leq \psi^{\prime \prime}\left(\frac{G}{M}\right)=\psi^{\prime \prime}\left(C_{p_{i}^{n_{i}}} \rtimes C_{2}\right) \leq \frac{13}{36},
$$

as desired. Consequently, $G$ is nilpotent.

Next we prove items a) and b). If $\psi^{\prime \prime}(G)>\frac{7}{16}$, then $G$ is nilpotent by c). Under the above notations, we have $G=C_{m} \times H$ and

$$
\psi^{\prime \prime}(H)=\psi^{\prime \prime}\left(\frac{G}{C_{m}}\right) \geq \psi^{\prime \prime}(G)>\frac{7}{16}
$$

implies that $H$ is cyclic by Lemma 2.2. Thus $G$ is cyclic. Similarly, if $\psi^{\prime \prime}(G)>\frac{27}{64}$, then one obtains $G=C_{m} \times H$, where either $H$ is cyclic or $H \cong C_{2} \times C_{2}$. Thus $G$ is abelian.

We prove now item d). We proceed by induction on $|G|$. Since cyclic-bysupersolvable groups are supersolvable, it suffices to show that $G$ contains a non-trivial cyclic normal subgroup $C$. Indeed, in this case we would have

$$
\psi^{\prime \prime}\left(\frac{G}{C}\right) \geq \psi^{\prime \prime}(G)>\frac{31}{144}
$$

and so $G / C$ would be supersolvable by the inductive hypothesis. It is clear that the condition $\psi^{\prime \prime}(G)>\frac{31}{144}$ implies that there exists $x \in G$ such that

$$
[G:\langle x\rangle]<\frac{144}{31} \text {, i.e. }[G:\langle x\rangle] \in\{1,2,3,4\} \text {. }
$$

Obviously, we can choose $C=\langle x\rangle$ for $[G:\langle x\rangle] \in\{1,2\}$. Assume that $[G:\langle x\rangle]=3$. If $\operatorname{Core}_{G}(\langle x\rangle) \neq 1$, then we can choose $C=\operatorname{Core}_{G}(\langle x\rangle)$, while if $\operatorname{Core}_{G}(\langle x\rangle)=1$ we infer that $G$ is supersolvable because it can be embedded in $S_{3}$. Assume now that $[G:\langle x\rangle]=4$. Again, if Core $_{G}(\langle x\rangle) \neq 1$, 
then we can choose $C=\operatorname{Core}_{G}(\langle x\rangle)$, while if $\operatorname{Core}_{G}(\langle x\rangle)=1$ we infer that $G$ can be embedded in $S_{4}$. Since $A_{4}$ and $S_{4}$ are the unique non-supersolvable subgroups of $S_{4}$ and

$$
\psi^{\prime \prime}\left(S_{4}\right)=\frac{67}{576}<\psi^{\prime \prime}\left(A_{4}\right)=\frac{31}{144}<\psi^{\prime \prime}(G),
$$

it follows that $G$ is supersolvable.

Finally, we prove item e). Similarly with d), it suffices to show that $G$ contains a non-trivial cyclic normal subgroup. The condition $\psi^{\prime \prime}(G)>\frac{211}{3600}$ implies that there exists $x \in G$ such that

$$
[G:\langle x\rangle] \leq 17
$$

If $[G:\langle x\rangle] \in\{16,17\}$, then the conclusion follows by Theorem 1.4. So, we can suppose that

$$
[G:\langle x\rangle] \leq 15
$$

If $|G| \geq 225$, then

$$
|\langle x\rangle| \geq \frac{225}{[G:\langle x\rangle]} \geq[G:\langle x\rangle]
$$

and therefore $\operatorname{Core}_{G}(\langle x\rangle)$ is a non-trivial cyclic normal subgroup of $G$ by Theorem 1.3. Suppose now that $|G|<225$ and that $G$ is non-solvable. Then one of the following holds:

(i) $|G|=60$ and $G \cong A_{5}$;

(ii) $|G|=120$ and $G \cong A_{5} \times C_{2}$ or $G \cong S_{5}$ or $G \cong \mathrm{SL}(2,5)$;

(iii) $|G|=168$ and $G \cong \operatorname{PSL}(2,7)$;

(iv) $|G|=180$ and $G \cong A_{5} \times C_{3}$.

If $G \cong A_{5} \times C_{n}$ with $n=2,3$, then Lemma $1.2,3$ ), leads to

$$
\psi^{\prime \prime}(G) \leq \psi^{\prime \prime}\left(A_{5}\right) \psi^{\prime \prime}\left(C_{n}\right)<\psi^{\prime \prime}\left(A_{5}\right),
$$

a contradiction. Using GAP, in the other cases we get

$$
\begin{aligned}
& \psi^{\prime \prime}\left(S_{5}\right)=\frac{471}{14400}<\frac{211}{3600} \\
& \psi^{\prime \prime}(\operatorname{SL}(2,5))=\frac{663}{14400}<\frac{211}{3600}, \\
& \psi^{\prime \prime}(\operatorname{PSL}(2,7))=\frac{715}{28224}<\frac{211}{3600},
\end{aligned}
$$

contradicting again the hypothesis.

The proof of Theorem 1.1 is now complete. 


\section{References}

[1] H. Amiri, S.M. Jafarian Amiri, I.M. Isaacs, Sums of element orders in finite groups, Comm. Algebra 37 (2009), 2978-2980.

[2] H. Amiri, S.M. Jafarian Amiri, Sums of element orders on finite groups of the same order, J. Algebra Appl. 10 (2) (2011), 187-190.

[3] S.M. Jafarian Amiri, Second maximum sum of element orders on finite nilpotent groups, Comm. Algebra 41 (6) (2013), 2055-2059.

[4] S.M. Jafarian Amiri, M. Amiri, Second maximum sum of element orders on finite groups, J. Pure Appl. Algebra 218 (3) (2014), 531-539.

[5] M. Baniasad Asad, B. Khosravi, A criterion for solvability of a finite group by the sum of element orders, J. Algebra 516 (2018), 115-124.

[6] I.M. Isaacs, Finite Group Theory, Amer. Math. Soc., Providence, Rhode Island, 2008.

[7] M. Herzog, P. Longobardi, M. Maj, An exact upper bound for sums of element orders in non-cyclic finite groups, J. Pure Appl. Algebra 222 (7) (2018), 1628-1642.

[8] M. Herzog, P. Longobardi, M. Maj, Two new criteria for solvability of finite groups in finite groups, J. Algebra 511 (2018), 215-226.

[9] M. Herzog, P. Longobardi, M. Maj, Sums of element orders in groups of order $2 m$ with $m$ odd, to appear in Comm. Algebra.

[10] M. Herzog, P. Longobardi, M. Maj, The second maximal groups with respect to the sum of element orders, arXiv:1901.09662.

[11] W.R. Scott, Group theory, Prentice-Hall, Inc., Englewood Cliffs, New Jersey, 1964.

[12] R. Shen, G. Chen, C. Wu, On groups with the second largest value of the sum of element orders, Comm. Algebra 43 (6) (2015), 2618-2631.

[13] M. Suzuki, Group theory, I, II, Springer Verlag, Berlin, 1982, 1986.

[14] M. Tărnăuceanu, A criterion for nilpotency of a finite group by the sum of element orders, submitted, arXiv:1903.09744. 
Marius Tărnăuceanu
Faculty of Mathematics
"Al.I. Cuza" University
Iaşi, Romania
e-mail: tarnauc@uaic.ro 